\title{
Place relative des essais cliniques comparatifs et des suivis de cohorte dans l'évaluation pré- et post-AMM des médicaments
}

\author{
Muriel Vray, ${ }_{1}^{1}$ Bernard Hamelin, ${ }^{2}$ Patrice Jaillon ${ }^{3}$ et les participants à la Table Ronde $n^{\circ} 2$ \\ de Giens $X X^{*}$ \\ 1 Unité d'Epidémiologie des Maladies Emergentes, Institut Pasteur, Paris, France \\ 2 Laboratoires AstraZeneca, Rueil-Malmaison, France \\ 3 Hôpital Saint-Antoine, Paris, France
}

\section{Résumé}

\begin{abstract}
La place relative des essais cliniques comparatifs et des études observationnelles (cohortes ou études castémoins) a été étudiée dans l'objectif d'évaluer l'efficacité, la tolérance et l'intérêt de santé publique d'un médicament. L'essai randomisé, comparatif, réalisé en double insu est la meilleure méthode pour estimer l'efficacité d'un traitement. Il permet l'estimation la moins biaisée et la plus robuste de la relation causale. Dans certaines situations et sous certains critères, des études observationnelles peuvent avoir valeur de preuve d'efficacité. Les études cliniques randomisées comparatives en pré- et post-AMM (autorisation de mise sur le marché) permettent d'identifier les effets indésirables plus rares et de les comparer au traitement de référence. Avant l'AMM, les données de sécurité regroupées des différents essais comparatifs peuvent permettre d'estimer des événements indésirables relativement fréquents et les sujets à risque. Cependant, l'étude observationnelle est plus adaptée pour évaluer la sécurité d'emploi dans les conditions réelles d'utilisation. Par définition, un médicament a un impact sur la santé des populations s'il permet d'améliorer directement ou indirectement son état de santé. Un médicament aura un intérêt majeur en termes de santé publique s’il réduit la mortalité ou la morbidité liée à cette maladie ou s'il améliore la qualité de vie des patients qui en sont atteints. Avant la commercialisation d'un produit, la modélisation est l'approche de choix pour quantifier l'impact attendu. En post-AMM, les essais pragmatiques et les études observationnelles sont les méthodes de référence pour définir la population rejointe, l'efficacité et la tolérance en situation réelle et l'intérêt en santé publique d'un médicament. En conclusion, l'essai clinique randomisé reste la référence pour l'évaluation de l'efficacité, l'étude observationnelle ayant une valeur confirmatoire. L'étude observationnelle est plus adaptée pour évaluer la sécurité d'emploi dans les conditions réelles d'utilisation, l'essai clinique ayant des indications limitées. Pour l'intérêt de santé publique, la modélisation est l'approche de choix en pré-AMM. En post-AMM, les essais pragmatiques et les études observationnelles sont les références.

Mots clés : méthodologie, essai randomisé, cohortes, évaluation pré- et post-AMM
\end{abstract}

Jusqu'à présent, l'essentiel de l'évaluation du rapport bénéfice/ risque d'un nouveau médicament reposait sur les résultats des essais cliniques contrôlés nécessaires pour obtenir l'autorisation de mise sur le marché (AMM) du médicament. Après l'AMM, et en dehors de rares études pharmacoépidémiologiques de cohortes ou de cas-témoins réalisées souvent à l'initiative du laboratoire pharmaceutique, le suivi du médicament était assuré par la noti- fication des effets indésirables par les professionnels de santé au système national de pharmacovigilance ou au service de pharmacovigilance de la firme concernée. Par ailleurs, l'Afssaps (Agence française de sécurité sanitaire des produits de santé) pouvait prendre l'initiative de déclencher des enquêtes nationales de pharmacovigilance avec un suivi essentiellement orienté par des objectifs de sécurité et de santé publique. En revanche, les notions

\footnotetext{
* Pour la liste des participants, voir en fin d'article.
} 
de définition de «population rejointe » par le médicament ou de vérification de la quantité d'effet thérapeutique apportée par le médicament dans la «vraie vie », c'est-à-dire après sa mise sur le marché, étaient peu évaluées.

A l'initiative de la Direction Générale de la Santé, le besoin d'étudier l'efficacité et la sécurité du nouveau médicament après qu'il ait été mis sur le marché est devenu une évidence scientifique, si ce n'est encore réglementaire. Le retrait récent du marché d'un anti-inflammatoire non stéroïdien antagoniste de la cyclo-oxygénase (COX)-2, le rofécoxib, à la suite de la mise en évidence d'une toxicité cardiovasculaire, vient d'illustrer ce besoin d'informations post-AMM. Il n'est plus contestable aujourd'hui d'observer que les populations de patients traités par un nouveau médicament diffèrent parfois profondément des populations de patients qui ont été inclus dans les essais cliniques pré-AMM et que les données d'efficacité/sécurité du médicament recueillies avant l'AMM ne sont pas directement transposables à l'utilisation du médicament après l'AMM.

C'est donc à juste titre que doivent être analysées et comparées les méthodologies utilisables en pré- et en post-AMM pour évaluer le véritable rapport bénéfice/risque d'un nouveau médicament. L'objectif de cette table ronde a été d'envisager la place relative des essais cliniques comparatifs et des suivis de cohortes dans l'évaluation pré- et post-AMM des médicaments.

\section{Mesure de l'efficacité}

\subsection{Les essais et les études}

L'essai clinique comparatif réalisé en double insu demeure la meilleure méthode pour mesurer l'efficacité d'un médicament. Le tirage au sort des traitements attribués aux patients et le maintien du double insu pendant l'essai constituent les meilleures « défenses » contre l'existence possible de biais qui viendraient brouiller les résultats de l'essai (biais de sélection des patients, d'attribution des traitements, de subjectivité dans l'interprétation des résultats, etc). Cet essai clinique, avec tirage au sort et double insu, permet l'estimation la moins biaisée et la plus robuste de l'efficacité du médicament. Il apporte le plus fort niveau de preuve de l'efficacité d'un médicament et constitue le fondement méthodologique essentiel des essais cliniques pré-AMM réalisés en phase III qui permettent de définir l'indication thérapeutique du médicament, la population cible du traitement, la posologie à respecter et les éventuelles précautions d'emploi ou contre-indications qui devront être respectées après l'AMM.

Les études observationnelles de cohortes, les études cas-témoins rétrospectives ou prospectives, les essais pragmatiques ouverts et avec tirage au sort doivent également trouver leur place dans l'évaluation de l'efficacité d'un médicament. Ces différents types d'études cliniques, jusqu'à présent réservées à la période post-AMM, peuvent, dans certaines conditions, apporter une preuve supplémentaire de l'efficacité du médicament.

\subsection{Critères de qualité}

Qu'il s'agisse d'essais comparatifs avec tirage au sort des médicaments ou d'études observationnelles, ces différentes méthodologies ne permettent une estimation de l'efficacité d'un médicament que si certains critères sont respectés.

- La méthodologie doit être correcte pour pouvoir extrapoler les résultats à la population des patients. Les biais possibles doivent pouvoir être identifiés pour être ensuite pris en compte dans l'analyse et dans l'interprétation des résultats. Le choix du médicament comparateur doit être justifié du point de vue pharmacologique.

- Dans les essais comparatifs, l'essai de supériorité permet une meilleure preuve d'efficacité que l'essai de non-infériorité. En effet, dans l'hypothèse de non-infériorité, la preuve d'efficacité est faite indirectement par rapport à un médicament de référence. La non-infériorité, et donc l'efficacité, est acceptée par rapport à une borne de non-infériorité définie $a$ priori.

- La bonne qualité de la réalisation des essais ou des études observationnelles, la taille de l'intervalle de confiance, la robustesse des résultats, sont autant de facteurs importants à considérer pour valider des résultats.

- L'importance de la différence observée dans un essai comparatif est un élément capital pour l'évaluation de la pertinence des résultats de l'essai. On sait notamment combien la seule évaluation de la diminution du risque relatif dans un essai comparatif est trompeuse pour les cliniciens qui ne réalisent pas toujours que l'évaluation de la diminution du risque absolu minimise considérablement l'intérêt réel du nouveau médicament par rapport au médicament de référence.

- Enfin, la reproductibilité des résultats observés dans différentes études est un gage de sécurité lorsque ces résultats sont convergents.

\subsection{Places respectives des essais comparatifs et des études observationnelles}

L'essai clinique comparatif réalisé avec tirage au sort et double insu apporte le niveau de preuve maximum quant à l'efficacité thérapeutique d'un médicament. Il est exceptionnel qu'une seule étude observationnelle de cohorte établisse un niveau de preuve suffisant pour valider l'enregistrement d'une nouvelle molécule 
ou d'une nouvelle indication. Le CPMP (Committee for Proprietary Medicinal Products) a établi les critères requis pour justifier de l'impossibilité de réaliser un essai comparatif en l'état actuel des connaissances :

- une maladie grave avec une probabilité nulle ou exceptionnelle d'amélioration spontanée ;

- une efficacité exceptionnelle avec des résultats individuels évidents ;

- un profil de sécurité documenté et acceptable ;

- l'absence d'alternative thérapeutique ;

- la recherche de données sur des témoins historiques.

Dans le cas d'un médicament avec une indication rare, il peut être utile, après la réalisation d'un essai comparatif, de mettre en place une étude de cohorte. C'est le cas par exemple de l'évaluation de l'efficacité d'un antibiotique dans une pathologie à germes multirésistants faisant suite à l'évaluation de son efficacité dans une pathologie infectieuse à germes plus courants. Un essai comparatif randomisé peut être accompagné d'une étude observationnelle non comparative ciblant, par exemple, les patients non éligibles pour l'essai du fait des critères de non-inclusion sur la gravité de la maladie. Une étude observationnelle, cohorte ou étude rétrospective cas-témoins, peut permettre d'identifier un effet (efficacité ou sécurité) intéressant du médicament qui pourra ensuite être confirmé par un essai comparatif randomisé. Enfin, et c'est la situation la plus fréquente, un essai comparatif randomisé peut être suivi d'une étude observationnelle de cohorte ou d'un essai pragmatique dans le but de confirmer l'efficacité du médicament lors d'un suivi à long terme et en conditions réelles d'utilisation.

\section{Mesure de la sécurité du médicament}

\subsection{En pré-autorisation de mise sur le marché (AMM) l'essai clinique est la méthode de référence pour évaluer la sécurité}

L'essai clinique randomisé contrôlé permet l'estimation la plus robuste d'une relation de cause à effet et la comparaison entre le traitement étudié et les médicaments comparateurs, en particulier pour les effets indésirables «fréquents » $(>1 \%)$ ou préalablement identifiés. Cependant, il présente plusieurs limitations importantes :

- Le schéma de l'étude est expérimental, du fait du tirage au sort de l'attribution des traitements, et ainsi ne tient pas compte de la préférence du prescripteur et du patient. L'essai clinique reflète donc mal la complexité des situations cliniques.
- Les critères d'éligibilité dans les essais sont souvent restrictifs afin de rendre la population évaluée la plus homogène possible. Ainsi l'extrapolation des données obtenues dans les essais n'est pas toujours possible pour la population générale, notamment en matière de sécurité.

- L'essai clinique manque souvent de puissance statistique pour détecter des événements indésirables rares $(<1 / 1000)$ ou très rares $(<1 / 10000)$.

Les données de tolérance recueillies lors des essais en préAMM sont regroupées afin d'explorer les bilans de sécurité en fonction du profil pharmacologique du médicament et des différents types de patients (critères démographiques, antécédents ...).

Regrouper les résultats des différents essais permet également, en augmentant la puissance statistique, de réaliser des analyses sur des sous-groupes de patients à risque. Cependant, ces méthodes d'analyse ne permettent pas toujours de détecter des événements rares. Lorsqu'un signal est détecté lors de l'évaluation initiale sur les données regroupées, il est alors important de mettre en place, dans le post-AMM, soit des essais cliniques dans les sous-groupes particuliers identifiés comme à risque soit des études observationnelles de type cas-témoins (statistiquement plus puissantes mais plus soumises à différents biais) afin de confirmer ou d'infirmer l'existence d'un risque potentiel pour le patient.

\subsection{En post-AMM, la pharmacovigilance et les études pharmacoépidémiologiques permettent de compléter l'évaluation initiale}

L'expérience récente a montré que la détermination complète des risques potentiels d'un médicament ne peut réellement émerger qu'après l'AMM ; soit par identification d'une série de cas grâce à la surveillance post-AMM des effets indésirables (pharmacovigilance), soit par la mise en place d'études spécifiques post-AMM (le plus souvent de type pharmacoépidémiologiques).

\subsection{Les données de pharmacovigilance}

Les données de pharmacovigilance permettent de contrôler la sécurité d'emploi des médicaments en condition réelle d'utilisation et de détecter un risque accru d'événements graves. Cependant, inadaptées pour estimer une incidence dans la population exposée au traitement (population rejointe), elles posent le problème de la qualité variable des informations recueillies et de leurs méthodes de recueil. Il s'agit d'un système d'alarme reposant sur l'identification de séries de cas qui est très performant pour détecter un signal. 


\subsubsection{Les études observationnelles}

Les études observationnelles sont définies par opposition aux essais cliniques contrôlés par le fait que le traitement attribué au sujet est délibérément choisi par le médecin. Elles permettent d'évaluer la sécurité du médicament, « dans la vraie vie », dans la population rejointe et dans les conditions réelles de son utilisation par le patient. On distingue plusieurs types d'études observationnelles :

- Les études de type «cohortes » qui mesurent la fréquence d'un événement et l'existence d'une association entre un facteur d'exposition (le médicament) et l'événement (l'effet indésirable).

- Les études cas-témoins qui mesurent uniquement l'existence d'une association.

Les risques de biais associés aux études observationnelles réalisées pour évaluer les effets d'un traitement ont été bien décrits dans la littérature médicale. Les résultats sont en partie limités du fait de l'existence de facteurs pronostiques non identifiés.

Cependant, en se rapprochant le plus possible de la routine clinique, ces études permettent une meilleure extrapolation des résultats à l'ensemble de la population traitée en vie réelle et, grâce à une puissance statistique accrue, du fait de la taille souvent très importante des échantillons, elles sont plus adaptées pour identifier et quantifier des effets indésirables rares ou très rares que la déclaration spontanée et l'essai contrôlé.

\section{Mesure de l'impact de santé publique}

Après qu'un médicament ait fait la preuve d'un rapport bénéfice/risque suffisant pour obtenir une AMM, une évaluation de type populationnelle est nécessaire pour évaluer son intérêt en santé publique.

Par définition, un médicament a un impact sur la santé des populations s'il permet d'améliorer directement ou indirectement son état de santé. L'impact de santé publique peut se définir par le nombre d'événements morbides ou de handicaps évités dans une population et sur une période de temps bien identifiées, et par les conséquences de la mise sur le marché de ce médicament sur le système de santé.

Un médicament aura un intérêt majeur en termes de santé publique s'il réduit la mortalité ou la morbidité liée à cette maladie ou s'il améliore la qualité de vie des patients qui en sont atteints.

\subsection{Contexte pré-AMM}

Avant la commercialisation du médicament, les principales questions qui se posent concernent la prévalence et l'incidence de la maladie en fonction des différents niveaux de gravité.

L'étape suivante consiste à définir la population cible idéale, c'est-à-dire celle qui devrait en théorie bénéficier de l'utilisation du médicament, et la population cible susceptible de l'utiliser en pratique. Il est aussi nécessaire de définir la population qui bénéficiera le plus du médicament, en tenant compte de la réalité des pratiques.

En fonction de ces éléments et de la quantité d'effet mesurée dans les études ayant constitué le dossier d'AMM, l'impact attendu de l'intérêt de santé publique pourra être estimé.

Hormis les essais de phase II/III du dossier d'AMM comparatifs, randomisés et réalisés si possible en double insu, les données permettant d'évaluer la population cible proviennent souvent d'études descriptives réalisées soit sur des cohortes soit à partir de panels sur les autres médicaments de la classe.

Des études ad hoc, type études observationnelles à partir d'échantillons représentatifs, peuvent répondre aux questions de définition de prévalence et de populations cibles.

L'évaluation de la quantité d'effet en situation réelle de prise en charge sur des critères de morbimortalité, la plupart du temps non documentés, est impossible, sauf dans le cas d'évaluation qui, dans la vraie vie, seront parfaitement extrapolables aux résultats observés dans les essais pré-AMM. Le recours à des modèles mathématiques peut permettre, par simulation, de quantifier l'impact attendu. Pour ce faire, on doit s'appuyer sur la connaissance du modèle d'effet du médicament - la relation qui lie la fréquence de l'événement clinique sous traitement à celle sans traitement et qui est spécifique à chaque médicament, pathologie et objectif thérapeutique - à condition que certains critères soient respectés : critère clinique disponible dans les études de phase III (et pas uniquement critère de substitution), accès à l'ensemble des données et description de l'algorithme utilisé.

Malheureusement, la plupart du temps on ne dispose pas encore de ces éléments lors de la mise sur le marché d'un médicament. Il faut alors essayer d'estimer le modèle d'effet à partir des résultats des essais en raisonnant en termes de classe thérapeutique, ce qui n'est évidemment pas possible dans le cas d'un médicament appartenant à une nouvelle classe thérapeutique. La prédiction de l'effet, à partir de ces modèles, sera d'autant meilleure qu'elle reposera sur des essais dans lesquels les populations sont hétérogènes et proches de la pratique. L'intérêt des modèles réside dans les analyses de sensibilité qui, en faisant varier les paramètres, permettent de quantifier les effets possibles. Toutefois, ces modèles, s'ils sont acceptables au 
moment de l'enregistrement, ne doivent pas dispenser les promoteurs de la mise en place d'études réalisées dans la vraie vie pour évaluer ce que les anglosaxons désignent par l' « effectiveness » et que nous pourrions traduire par l' « intérêt »du médicament en situation réelle.

En fonction de la pathologie, des médicaments et du mode de prise en charge des patients, différentes méthodes pourront être envisagées.

\subsection{Contexte post-AMM}

Lorsque le médicament est sur le marché, de nombreuses questions restent posées comme l'efficacité et la tolérance en situation pratique de prescription mais aussi des questions relatives aux habitudes des patients, des prescripteurs.

L'identification de la véritable population rejointe, c'est-àdire celle effectivement traitée par le médicament, qui peut différer de la population cible visée par les indications officielles accordées à ce médicament, est une question majeure de santé publique.

Les études visant à évaluer le bon usage du médicament ou encore son impact sur la prise en charge de la pathologie ou l'identification des facteurs prédictifs d'une bonne réponse ou d'une bonne tolérance devront aussi être mises en place.

Pour répondre à ces différentes questions, on est amené à choisir entre une approche observationnelle ou une approche plus expérimentale de type « essai pragmatique ».

Dans une approche observationnelle, en comparant des cohortes de sujets traités par différents médicaments, on ne fait qu'observer une population ou un phénomène sans intervenir ou en intervenant au minimum sur le cours naturel des choses. On peut penser que ces études ne modifieront pas trop la réalité. En pratique, il est toutefois illusoire de penser qu'une étude ne modifie en rien le comportement des patients et des prescripteurs alors qu'ils se savent observés. Dans ce contexte d'études observationnelles, on peut aussi s'interroger sur l'approche prospective ou rétrospective du recueil de l'information. Si l'approche prospective dans laquelle on suit les sujets traités jusqu'à l'apparition de l'événement cherché permet, en théorie, d'avoir moins de données manquantes elle peut davantage modifier la réalité de la prise en charge. A l'inverse, avec une approche rétrospective, l'effet (ou l'exposition) recherchée sera plus soumise aux biais de "mémorisation », conduisant à une surestimation de l'exposition chez les cas.

Les résultats observés dans ces études observationnelles devront tenir compte des biais dus à la représentativité de l'échantillon étudié, aux facteurs de confusion et aux sujets per- dus de vue souvent en nombre important. Des analyses avec ajustement sur les facteurs de confusion devront être réalisées.

A l'opposé, une approche plus expérimentale consiste à faire un essai thérapeutique avec tirage au sort de l'attribution des traitements ou des stratégies thérapeutiques. Cette option séduisante a priori car elle permet de « contrôler » le biais de non-comparabilité des groupes à l'inclusion, ne représente pas tout à fait la réalité, même si les critères d'inclusion des patients, le suivi et les critères d'évaluation, beaucoup plus proches de la « vraie vie », diffèrent largement de ceux retenus dans les essais de phase III. En effet, on retrouve le risque de modification du comportement des médecins et des patients qui se savent « suivis ». On peut aussi penser que certains patients/médecins refuseront de participer à une expérience dans laquelle les patients recevront un traitement par tirage au sort, même s'il n'existe aucun argument scientifique à ce qu'ils soient traités par l'un ou l'autre des traitements à l'étude.

L'estimation de l'intérêt de santé publique s'appuie donc principalement sur des études de cohortes ou sur des essais de type pragmatique.

\section{Conclusion}

L'essai clinique randomisé reste la référence pour l'évaluation de l'efficacité, l'étude observationnelle a une valeur complémentaire. Dans certaines situations et sous certains critères, des études observationnelles peuvent avoir valeur de preuve d'efficacité.

L'étude observationnelle est plus adaptée pour évaluer la sécurité d'emploi dans les conditions réelles d'utilisation, l'essai clinique a des indications limitées. Les résultats des essais contrôlés réalisés en double insu sur les données regroupées permettent d'identifier des événements relativement fréquents et les sujets à risque.

Pour évaluer l'intérêt de santé publique d'un médicament, la modélisation est l'approche de choix en pré-AMM. Les données qui constituent le dossier d'AMM permettent, souvent, au mieux d'estimer un intérêt de santé public attendu.

En post-AMM, les essais pragmatiques et les études observationnelles sont les références pour évaluer l'impact d'un médicament sur la population traitée.

\section{Participants}

M. Andréjak (CHU, Amiens), R. Benamouzig (Hôpital Avicenne, Bobigny), J.-P. Boissel (Faculté de Médecine, Lyon), C. Bremard-Oury (Aventis, Paris), C. Brun-Strang (Novartis, Rueil-Malmaison), E. Caulin (Aventis, Paris), D. Cellier (Merck Santé, Lyon), D. Costagliola (INSERM, Paris), V. Daurat (AP-HP DRRC, Paris), A. El Hasnaoui (GSK, Marly le Roi), 
J.-M. Goehrs (Merck Sharp \& Dohme-Chibret, Paris), B. Hamelin (AstraZeneca, Rueil-Malmaison), P. Jaillon (Hôpital St-Antoine, Paris), C. Jouan-Flahault (LEEM, Paris), J.-L. Montastruc (Faculté de Médecine, Toulouse), N. Moore (Hôpital Pellegrin, Bordeaux), A. Puech (Sanofi Synthelabo, Chilly-Mazarin), M. Ricatte (CNAMTS, Paris), P. Ricordeau (CNAMTS, Paris), M.-H. Rodde-Dunet (CNAMTS, Paris), E. Rouffiac (Abbott, Rungis), T. Simon (Hôpital St-Antoine, Paris), P. Simon (Sanofi Recherche, Paris), A.
Spriet (Spriet Conseil, Paris), E. Van Ganse (CHU, Lyon), P. Velicitat (Boehringer-Ingelheim, Reims), M. Vray (Institut Pasteur, Paris).

Correspondance et offprints : Bernard Hamelin, Laboratoire AstraZeneca, 1 place Renault, 92844 Rueil-Malmaison Cedex, France.

E-mail : bernard.hamelin@astrazeneca.com 